\title{
L'ipertrofia ventricolare sinistra nei pazienti affetti da malattia renale cronica
}

\section{Luca Di Lullo', Fulvio Floccari ${ }^{2}$, Rodolfo Rivera ${ }^{3}$, Antonio De Pascalis ${ }^{4}$, Vincenzo Barbera ${ }^{1}$, Moreno Malaguti ${ }^{2}$, Alberto Santoboni ${ }^{1}$}

\author{
${ }^{1}$ U.O.C. Nefrologia e Dialisi, Ospedale L. Parodi-Delfino, Colleferro (RM) \\ ${ }^{2}$ U.O.C. Nefrologia e Dialisi, Ospedale San Paolo, Civitavecchia (RM) \\ ${ }^{3}$ Divisione di Nefrologia, Ospedale S. Gerardo, Monza \\ ${ }^{4}$ U.O.C. Nefrologia, Dialisi e Trapianto, Ospedale V. Fazzi, Lecce
}

\begin{abstract}
LEFT VENTRICUL AR HYPERTROPHY IN PATIENTS WITH CHRONIC KIDNEY DISEASE
Abstract. Cardiovascular diseases such as coronary artery disease, congestive heart failure, arrhythmias, and sudden cardiac death represent main causes of morbidity and mortality in patients with chronic kidney disease (CKD). Their pathogenesis relates to the close linkage between heart and kidneys and involves both traditional and non-traditional risk factors. According to the well-established classification of cardio-renal syndrome, the cardiovascular involvement in chronic kidney disease is known as "type 4 cardiorenal syndrome" (chronic renocardiac syndrome).

Uremic cardiopathy is mainly characterized by both left ventricular systolic and diastolic impairment, often associated to right heart dysfunction due to the presence of a vascular access for hemodialysis.

The typical clinical picture is represented by left ventricular hypertrophy (LVH), the pathogenesis of which is multifactorial and closely linked to elevated blood pressure, vascular stiffness and atherosclerosis.

The diagnosis is mainly made by ultrasound (2D and 3D echocardiography) and cardiac magnetic resonance imaging (CMRI), although echocardiography is most widely employed since it is non-invasive and cheaper than CMRI.

The following chapter provides an overview of the epidemiology, pathophysiology, diagnosis, and treatment of left ventricular hypertrophy in CKD patients.
\end{abstract}

Key words: Cardiovascular disease, Type 4 cardiorenal syndrome, Left ventricular hypertrophy, Echocardiography

Conflict of interest: None.

Financial support: None.

Accettato: 10 Agosto 2014

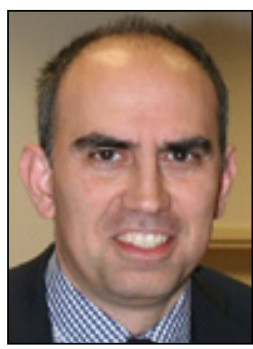

Luca Di Lullo

\section{Introduzione}

Le patologie cardiovascolari (sindrome coronarica acuta, cardiopatia ischemica cronica, scompenso cardiaco, aritmie, morte cardiaca improvvisa) rappresentano il principale fattore di mortalità e morbidità nei pazienti affetti da malattia renale cronica (CKD).

La presenza di questo "overlap" tra malattia renale e patologia cardiovascolare ha portato, negli ultimi anni, a definire il concetto di "Sindrome cardio-renale" e, nell'ambito di quest'ultima, l'interessamento cardiaco in corso di malattia renale cronica viene indicato con il termine di Sindrome cardio-renale di tipo 4 (la cosiddetta sindrome cardio-renale di tipo cronico reno-cardiaca).
Con il termine di Sindrome cardio-renale (SCR), infatti, si intende un ampio spettro di patologie caratterizzato dal coinvolgimento, in tempi successivi o in simultanea, di apparato cardiovascolare e rene.

Un'apposita Consensus Conference ha classificato la SCR in 5 sottotipi che, a loro volta, possono essere schematicamente divisi in sindromi cardio-renali (tipo 1 e tipo 3 ) e renocardiache (tipo 2 e tipo 4), entrambe sulla base del primum movens, e, a loro volta, differenziate in acute e croniche a seconda della modalità d'esordio della patologia (Tab. I) (1). Il tipo 5 di SCR racchiude invece tutte quelle condizioni nelle quali la SCR si sviluppa nell'ambito di patologie sistemiche che arrivano, in tempi più o meno lunghi, a interessare l'asse cardio-renale.

Come verrà poi ampiamente trattato nelle sezioni che seguiranno, la condizione di ipertrofia ventricolare sinistra rappresenta 
TABELLA I - CLASSIFICAZIONE DELLA SINDROME CARDIO-RENALE

\begin{tabular}{|c|c|c|c|}
\hline Tipo & Denominazione & Descrizione & Esempio \\
\hline 1 & Cardio-renale acuta & Insufficienza cardiaca acuta che conduce a IRA & $\begin{array}{l}\text { Sindrome coronarica acuta che porta a scompenso } \\
\text { cardiaco acuto e insufficienza renale }\end{array}$ \\
\hline 2 & Cardio-renale cronica & $\begin{array}{l}\text { Scompenso cardiaco cronico che conduce a } \\
\text { insufficienza renale }\end{array}$ & Scompenso cardiaco cronico \\
\hline 3 & Nefrocardiaca acuta & IRA che conduce a insufficienza cardiaca acuta & Cardiomiopatia uremica secondaria a IRA \\
\hline 4 & Nefrocardiaca cronica & IRC che conduce a insufficienza cardiaca & $\begin{array}{l}\text { Ipertrofia ventricolare sinistra e scompenso cardiaco } \\
\text { diastolico secondari a insufficienza renale }\end{array}$ \\
\hline 5 & Secondaria & $\begin{array}{l}\text { Patologie sistemiche che comportano insufficienza } \\
\text { cardiaca e insufficienza renale }\end{array}$ & Shock settico, vasculiti, diabete mellito \\
\hline
\end{tabular}

uno degli elementi chiave nella cosiddetta cardiopatia uremica (SCR di tipo 4, cronica reno-cardiaca).

Le complicanze di natura cardiovascolare possono essere riscontrate sin dai primi stadi di malattia renale cronica, indipendentemente dai livelli del filtrato glomerulare.

\section{Epidemiologia}

Come già anticipato nel paragrafo introduttivo, gli eventi cardiaci maggiori sono responsabili di quasi la metà dei decessi nei pazienti affetti da malattia renale cronica (2).

Il coinvolgimento cardiaco è presente sin dai primi stadi di CKD ed è legato in parte all'età media della popolazione che arriva alla diagnosi della malattia renale stessa e, in parte, all'elevata incidenza di diabete mellito, dislipidemia e ipertensione arteriosa tra i pazienti affetti da CKD (3).

Lo studio HEMO ha evidenziato in maniera abbastanza chiara l'elevata prevalenza (pari a circa $1^{\prime} 80 \%$ ) di patologia cardiovascolare nei pazienti emodializzati in relazione all'età, alla prevalenza di diabete mellito e all'età dialitica; la maggior parte dei pazienti arruolati nello studio, inoltre, era ricoverata per sindrome coronarica acuta.

I pazienti affetti da malattia renale cronica agli stadi I-IV presentano un minor grado di coinvolgimento cardiovascolare rispetto ai pazienti in trattamento dialitico (emodialisi o dialisi peritoneale); la diagnosi di malattia cardiovascolare si palesa con maggiori probabilità quando il valore del filtrato glomerulare (GFR) scende al di sotto di $60 \mathrm{~mL} / \mathrm{min} / 1.73 \mathrm{~m}^{2}$ (4-6).

Una metanalisi di Tonelli condotta su un campione di oltre 1 milione di pazienti ha permesso di riscontrare tassi più elevati di mortalità per tutte le cause in diretta correlazione con la caduta dei livelli di GFR (7).

Il rischio cardiovascolare diventa particolarmente evidente nei pazienti con CKD agli stadi IIIb-IV e in coloro i quali sono sottoposti a trattamento dialitico (8).

Il rischio di eventi cardiovascolari sfavorevoli, se paragonato a una popolazione di controllo con valori normali di GFR, si presenta più elevato del $43 \%$ nei pazienti con valori di GFR compresi nell'intervallo $45-59 \mathrm{~mL} / \mathrm{min} / 1.73 \mathrm{~m}^{2}$ e del $343 \%$ in coloro i quali presentano un valore di GFR inferiore a $15 \mathrm{~mL} /$ $\min / 1.73 \mathrm{~m}^{2}(8)$.
I pazienti affetti da CKD allo stadio V, ma non ancora sottoposti a trattamento sostitutivo della funzione renale, presentano tassi di mortalità cardiovascolare simili ai pazienti sottoposti a terapia dialitica $(9,10)$, tassi confermati anche dagli studi KEEP (Kidney Early Evaluation Program) e CRIC (Chronic Renal Insufficiency Cohort) $(11,12)$.

Quadri di ipertrofia ventricolare sinistra ed eventi cardiovascolari sono presenti anche in stadi precoci di malattia renale, come evidenziato nello studio ARIC (Atherosclerosis Risk in Communities), uno studio di popolazione che ha evidenziato la presenza di un incremento di IVS e scompenso cardiaco congestizio in pazienti con valori di GFR inferiori a $60 \mathrm{~mL} /$ $\min / 1.73 \mathrm{~m}^{2}(13)$.

La prevalenza di IVS varia, nelle diverse casistiche, dal $16 \%$ al $31 \%$ nei pazienti che presentano valori di GFR superiori a $30 \mathrm{~mL} / \mathrm{min}$, per passare a percentuali del $60-75 \%$ nei pazienti con CKD agli stadi IV-V e arrivare a tassi di prevalenza del $90 \%$ nei pazienti che iniziano il trattamento dialitico (14).

Foley ha evidenziato che il $74 \%$ dei pazienti incidenti in emodialisi presenta dati ecocardiografici compatibili con IVS e il $30 \%$ di essi presenta un concomitante quadro di insufficienza cardiaca $(15,16)$.

\section{Ipertrofia ventricolare sinistra in corso di malattia renale cronica: le basi fisiopatologiche}

Anomalie ecocardiografiche (riduzione della frazione di eiezione, aumento dei diametri e dei volumi telesistolico e telediastolico del ventricolo sinistro) sono di comune riscontro sin dai primi stadi di CKD.

I fattori patogenetici coinvolti nella genesi dell'ipertrofia ventricolare sinistra in corso di CKD sono stati divisi in tre categorie fondamentali (17-19):

- fattori correlati al post-carico;

- fattori correlati al pre-carico;

- fattori non collegati al post-carico e al pre-carico.

I primi sono sostanzialmente rappresentati dall'aumento delle resistenze vascolari periferiche, dall'aumento della pressione arteriosa e dalla riduzione della compliance vascolare dei 
grossi vasi, collegata, in parte, ai fenomeni di calcificazione della parete vasale (17-20); il risultato finale è costituito dall'ipertrofia delle cellule miocardiche e dal rimodellamento concentrico del ventricolo sinistro, che si accompagna, spesso, all'attivazione del sistema renina-angiotensina intracardiaco $(19,21)$.

Nella genesi dell'IVS sembrano, inoltre, essere coinvolti anche lo stress ossidativo e l'attività dell'enzima xantina-ossidasi (22), così come l'attivazione della via enzimatica della 5-fosfodiesterasi, come evidenziato dagli effetti farmacologici esercitati dal sildenafil in grado di rallentare la progressione dell'IVS (23).

Parlando dei fattori patogenetici collegati al pre-carico, c'è da segnalare il ruolo giocato dall'aumento della volemia intravascolare (da ritenzione di acqua e sodio), dall'anemia secondaria e dall'eventuale presenza di una fistola artero-venosa. Tutti questi fattori concorrono all'ispessimento delle cellule miocardiche e al rimodellamento eccentrico o asimmetrico del ventricolo sinistro $(14,24,25)$.

Va comunque sottolineato come i fattori patogenetici legati sia al pre-carico che al post-carico agiscano con effetti sinergici e additivi.

Il risultato del rimodellamento ventricolare sinistro in termini di ipertrofia tissutale comporta l'induzione di vie metaboliche che conducono all'attivazione di segnali cellulari pro-apoptotici da un lato e di vie metaboliche in grado di portare alla produzione ridondante di matrice extracellulare dall'altro, fino a giungere alla fibrosi miocardica $(26,27)$.

Una condizione consolidata di fibrosi miocardica determina una progressiva disfunzione della contrattilità miocardica, che conduce prima alla disfunzione sisto-diastolica del ventricolo sinistro, quindi a quadri di cardiomiopatia dilatativa e scompenso cardiaco congestizio (28).

La presenza di un grado elevato di fibrosi miocardica, allo stesso tempo, comporta delle alterazioni a carico di contrattilità ed eccitabilità delle cellule miocardiche con conseguente aumento della possibilità di andare incontro ad aritmie da rientro (17).

Anche l'attivazione del sistema renina-angiotensina promuove la formazione di tessuto fibrotico attraverso il potenziamento di segnali intracellulari in grado di promuovere la sintesi di TGF (transforming growth factor) (21).

Alla genesi dell'IVS può, inoltre, contribuire il deficit di ferro e/o di eritropoietina (29) o di vitamina D (30).

La terapia con agenti calciomimetici sembra essere in grado di indurre una regressione della percentuale di tessuto fibrotico senza influenzare la massa ventricolare sinistra (31).

La stessa presenza di una fistola artero-venosa può contribuire in maniera determinante all'insorgenza e al mantenimento di un quadro di IVS a causa dell'aumento del post-carico e, conseguentemente, del lavoro cardiaco (14).

Un'altra problematica rilevante in termini fisiopatologici è quello della rigidità (stiffness) delle pareti arteriose, causata dall'aumentata produzione e deposizione di fibre collagene, la quale, determinando un grado variabile di vasocostrizione, comporta un aumento delle resistenze vascolari periferiche.

Tale rigidità dei vasi arteriosi può, inoltre, essere aggravata anche dalla presenza di elevate concentrazioni di sodio plasmatico (al di sopra di $135 \mathrm{mMol} / \mathrm{L}$ ), le quali danneggiano direttamente l'endotelio vasale e, secondariamente, inibiscono il rilascio di ossido nitrico (32).

Agendo sui livelli di sodio plasmatico nel corso delle sessioni emodialitiche, si possono produrre effetti positivi sui livelli di pressione arteriosa sistemica e sulla compliance del ventricolo sinistro (33).

Studi recenti hanno concentrato l'attenzione su biomarcatori già coinvolti in altri processi patogenetici ma mai considerati fattori degni di attenzione in tema di insorgenza di IVS.

Un rappresentante della categoria è costituito dal Fibroblast Growth Factor-23 (FGF23), membro della famiglia dei fattori di crescita dei fibroblasti, coinvolto in maniera diretta nella patogenesi della malattia ossea correlata alla CKD (CKD-MBD), nonché nella patogenesi dell'iperparatiroidismo secondario (34).

La patogenesi della CKD-MBD è stata da sempre ascritta a un deficit di 1.25-Diidrossivitamina D $\left(1.25(\mathrm{OH})_{2} \mathrm{D}\right)$, con conseguente aumento dei livelli sierici di ormone paratiroideo (PTH) e squilibrio nei livelli sierici di calcio e fosforo $(34,35)$.

Il deficit di vitamina $\mathrm{D}$, associato a iperparatiroidismo secondario e a iperfosforemia, viene ora considerato come un fattore in grado di contribuire all'aumento del rischio cardiovascolare nei pazienti affetti da CKD (36).

La cascata di eventi appena descritta è stata in parte messa in discussione dalla scoperta del ruolo giocato dal FGF23 nella fisiopatologia dell'iperparatiroidismo secondario. Allo stato attuale, il FGF23 risulta essere il primo biomarcatore a mostrare delle anomalie di concentrazione nei pazienti affetti da CKD-MBD (37) e i suoi livelli plasmatici aumentano prima ancora che si verifichino delle alterazioni nei livelli sierici di PTH, $1.25(\mathrm{OH})_{2} \mathrm{D}$ e fosfato (38).

Il FGF23 è coinvolto in regolazione, crescita e differenziazione dei miocardiociti, possedendo delle proprietà di tipo paracrino a causa della sua azione di tipo fosfaturico; è in grado di bloccare la sintesi di vitamina D3 e inibisce il riassorbimento del fosforo a livello del tubulo prossimale (39).

I livelli sierici di FGF23 aumentano gradualmente e vanno di pari passo con la riduzione della funzione renale (40).

I primi dati riguardanti la correlazione tra livelli sierici di FGF23 e mortalità sono del 2008, grazie allo studio di Gutierrez, che ha valutato i livelli plasmatici di FGF23 in 400 pazienti incidenti in emodialisi, concludendo come concentrazioni elevate di FGF23 siano indipendentemente associate a un incremento del rischio cardiovascolare nel primo anno di trattamento dialitico (40).

I risultati delle succitate osservazioni sono stati successivamente confermati in due studi di coorte in pazienti affetti da CKD (ma non ancora sottoposti a trattamento dialitico), i quali hanno entrambi documentato una stretta correlazione tra livelli sierici di FGF23 e aumentato rischio di eventi cardiovascolari, come infarto del miocardio e vasculopatia obliterante degli arti inferiori $(41,42)$.

I risultati di altri trial clinici hanno, inoltre, indicato una chiara correlazione tra livelli plasmatici di FGF23 e quadri ecocardiografici indicativi di IVS; in uno di questi trial, 124 pazienti emodializzati hanno evidenziato livelli di FGF23 indipendentemente associati alla presenza di IVS (43).

Un secondo trial clinico, condotto su 162 pazienti affetti da 
CKD agli stadi IV-V, ha evidenziato come i livelli di FGF23 siano indipendentemente associati alla massa ventricolare sinistra (44).

Livelli più elevati di FGF23 sembrano, inoltre, associati a una riduzione della frazione di eiezione, a un aumento della massa ventricolare sinistra e a una maggiore prevalenza di IVS, prevalentemente di tipo asimmetrico (32).

Diversi studi clinici hanno, nell'ultima decade, posto l'accento sulle relazioni intercorrenti tra livelli di vitamina $\mathrm{D}$, infiammazione cronica, calcificazioni vascolari e tassi di sopravvivenza $(45,46)$.

La vitamina $\mathrm{D}$ è, inoltre, coinvolta nella regolazione del sistema immunitario e di quello cardiovascolare e dell'apparato endocrino, mediante l'attivazione del recettore, ad alta affinità, per la vitamina $\mathrm{D}$ (VDR).

Alla luce del ruolo rilevante giocato dalla vitamina $\mathrm{D}$ nella patogenesi di diverse cardiomiopatie, di recente è stato studiato il rapporto potenzialmente esistente tra genesi dell'IVS e polimorfismo del VDR.

Il polimorfismo del gene BsmI è risultato coinvolto nella genesi dell'IVS nei pazienti con malattia renale cronica terminale $(47,48)$ e risulta indipendentemente correlato con il grado di progressione dell'IVS nei pazienti affetti da CKD agli stadi IIIb-IV e in coloro i quali sono sottoposti a trattamento dialitico (47).

La presenza di una variante mutata del gene BsmI del VDR nei pazienti con malattia renale cronica terminale è stata proposta come marker di disfunzione della via metabolica della vitamina $\mathrm{D}$, in grado di incidere sull'incremento della massa ventricolare sinistra (48).

\section{Conseguenze cliniche dell'aumento della massa ventricolare sinistra e della fibrosi miocardica in corso di CKD ed ESRD}

Come conseguenza dello sviluppo di IVS e di un'evoluzione verso il quadro di fibrosi miocardica, si assiste a una diminuzione della densità capillare del tessuto miocardico con esito finale nella disfunzione sisto-diastolica del ventricolo sinistro (dovuta ad anomalie via via crescenti della fase di riempimento rapido del ventricolo sinistro) e in disturbi della conduzione intraventricolare; il quadro clinico è ulteriormente aggravato da una successiva evoluzione verso un quadro di cardiomiopatia dilatativa che, solo in parte, viene mitigato da una progressiva ulteriore fase di ipertrofia compensatoria del ventricolo sinistro (cardiopatia uremica) (18).

Il grado di severità e la persistenza di un quadro di IVS sono significativamente associati al rischio di mortalità e agli eventi cardiovascolari in corso di CKD (49); è stato, inoltre, evidenziato, come la riduzione del $10 \%$ della massa ventricolare sinistra comporti un decremento del $28 \%$ del rischio di mortalità in pazienti sottoposti a trattamento emodialitico (50). Fattori predittivi di regressione dell'IVS sono sicuramente rappresentati dal controllo ottimale della pressione arteriosa sistemica, da una diminuzione della pulse-wave velocity e dall'aumento dei livelli sierici di emoglobina (50).

A far da contraltare a quanto appena detto, va riportato come, nonostante un controllo ottimale dei fattori di rischio (pressione arteriosa sistemica, malattia coronarica cronica e diabete mellito), i pazienti affetti da ESRD presentino un rischio maggiore, rispetto alla popolazione controllo, di andare incontro a morte cardiaca improvvisa (SCD), a testimonianza del fatto che altri fattori (diversi dalla malattia coronarica) devono essere chiamati in causa (IVS e fibrosi miocardica).

La presenza di un quadro di IVS raddoppia il rischio di SCD nel gruppo di pazienti arruolati nel trial clinico 4D (51); nello stesso trial clinico si è evidenziato come i livelli plasmatici di NT-pro-BNP fossero legati all'incidenza di SCD, così come la presenza di alterazioni metaboliche (iperfosfatemia, iperparatiroidismo secondario) o elettrolitiche (ipo-iperkaliemia) e l'iperattività del sistema nervoso simpatico. Nell'ambito dello studio 4D si è anche documentata l'associazione di IVS e aumento dei livelli sierici di pro-BNP con la sindrome del QT lungo e con un quadro di sindrome coronarica acuta (51).

Reperti autoptici in pazienti affetti da CKD evidenziano la presenza di una diffusa fibrosi miocardica con pattern assolutamente peculiare rispetto a quello documentato in pazienti affetti da ipertensione arteriosa severa ma esenti da CKD (52). La presenza di IVS risulta essere fortemente associata a un outcome cardiovascolare sfavorevole sia in presenza che in assenza di una malattia renale.

Studi longitudinali di coorte che si sono posti lo scopo di documentare la storia naturale dell'incremento della massa ventricolare sinistra in corso di CKD hanno evidenziato come la prevalenza di IVS aumenti al diminuire della funzionalità renale (53).

La presenza di ipertensione arteriosa (prevalentemente sistolica) e di un'elevata pressione pulsatoria è fortemente associata al quadro di IVS nei pazienti affetti da CKD, suggerendo come l'aumento di volume plasmatico e l'aumentata rigidità delle pareti arteriose giochino un ruolo nella genesi dell'IVS ancora prima che i pazienti inizino il trattamento emodialitico (53).

Risposte potenzialmente positive a una riduzione della massa ventricolare sinistra sono associate a presenza di età inferiore a 50 anni (indipendentemente dal sesso), presenza di livelli più bassi di pressione pulsatoria e valori più elevati di GFR (54).

Come già in parte anticipato, un certo grado di riduzione del grado di IVS può essere ottenuto con un controllo adeguato del bilancio idrico e della pressione arteriosa, insieme a un controllo ottimale dei livelli di emoglobina sierica (55).

Uno studio di Foley ha riscontrato una diminuzione della massa ventricolare sinistra e un miglioramento della funzione sistolica del ventricolo sinistro dopo un anno dall'inizio del trattamento dialitico, il tutto associato a una riduzione dell'incidenza di scompenso cardiaco ma non degli eventi ischemici e/o di morte cardiaca improvvisa (56).

Covic ha documentato la riduzione della massa ventricolare sinistra in pazienti sottoposti a trattamento emodialitico in concomitanza con un miglioramento dei parametri emocromocitometrici e dei livelli sierici di calcio e fosforo (57).

Altri Autori hanno riscontrato la presenza di ipertensione arteriosa (prevalentemente diastolica), tachicardia e aumentato rischio di accidenti cerebrovascolari nei pazienti con iperfo- 
sfatemia rispetto ai pazienti normofosfatemici (58).

La presenza di elevati livelli di fosfato sierico è associata a segni di disfunzione diastolica del ventricolo sinistro e a fibrosi miocardica ed è in grado di favorire l'insorgenza di IVS; il trattamento farmacologico dell'iperfosforemia potrebbe, quindi, diventare un elemento importante nel "management" di questo gruppo di pazienti (59).

\section{Elementi chiave nel trattamento dell'ipertrofia ventricolare sinistra nei pazienti affetti da CKD ed ESRD}

I punti chiave del trattamento dell'IVS nei pazienti affetti da CKD risiedono principalmente nel controllo pressorio, nella corretta gestione dell'anemia secondaria e nel trattamento ottimale dell'iperfosforemia e delle complicanze dell'iperparatiroidismo secondario.

L'impatto della terapia con eritropoietina (EPO) sul quadro di IVS nei pazienti affetti da CKD è stato esaminato in diversi trial clinici randomizzati, ma molti di essi hanno fallito nel dimostrare se l'eventuale correzione dello stato anemico comportasse una corrispettiva riduzione della massa ventricolare sinistra.

Parfrey ha effettuato una metanalisi di 15 trial clinici, dimostrando come la massa ventricolare sinistra si riducesse, grazie alla correzione dell'anemia secondaria, soltanto in quei pazienti che partivano da valori di emoglobina più bassi (al di sotto di $10 \mathrm{~g} / \mathrm{dL}$ ) e trattati allo scopo di ottenere livelli target di emoglobina più bassi (al di sotto di $12 \mathrm{~g} / \mathrm{dL}$ ) (60).

Chen et al. hanno paragonato gli effetti dell'EPO alfa e della darbopoetina alfa sulla regressione dell'IVS in pazienti affetti da CKD e hanno riscontrato come entrambi i farmaci fossero parimenti in grado di diminuire la massa ventricolare sinistra (61).

La correzione di un quadro anemico severo $(\mathrm{Hb}<10 \mathrm{~g} / \mathrm{dL})$ con EPO sembra essere in grado di controllare gli effetti lesivi dell'aumento della massa ventricolare sinistra; tale effetto della terapia con EPO non sembra evidente allorché la terapia viene impiegata in soggetti che partono da livelli più elevati di $\mathrm{Hb}$ sierica e nei quali viene effettuata una correzione più decisa portando i livelli emoglobinici oltre $i$ $12 \mathrm{~g} / \mathrm{dL}(62)$.

Altro provvedimento terapeutico di primaria importanza riguarda il mantenimento dei livelli di pressione arteriosa sistolica al di sotto dei $140 \mathrm{mmHg}$; tale azione produce effetti assolutamente favorevoli sull'evoluzione dell'IVS nei pazienti con CKD ed ESRD (63-65), specialmente se accompagnata da un'adeguata politica di controllo del volume extracellulare (66).

Un altro punto cardine del controllo dell'IVS potrebbe risiedere nella correzione dei disturbi correlati all'iperparatiroidismo secondario e, in particolare, ci si riferisce al controllo dei livelli della fosforemia; sfortunatamente, allo stato attuale, non vi sono trial clinici che possano confermare la tesi appena esposta (67).

I pazienti che sono in terapia con vitamina $\mathrm{D}$ evidenziano, comunque, una frequenza inferiore di eventi cardiovascolari, come testimoniato da alcuni studi osservazionali (67), mentre altri trial hanno documentato come la mancata regressione dell'IVS si associ a livelli più elevati di PTH $(>500 \mathrm{pg} / \mathrm{mL})(57)$.

Un altro punto chiave del trattamento dell'IVS consiste indubbiamente nella gestione del trattamento emodialitico e degli scambi in caso di pazienti in trattamento mediante dialisi peritoneale.

Uno schema che preveda sessioni di emodialisi maggiormente ravvicinate o di durata maggiore (incluse la dialisi quotidiana breve e la dialisi notturna lunga) è stato più volte suggerito e i primi dati (studi osservazionali) pervenuti sono assolutamente confortanti, vista la minore incidenza di IVS nei pazienti sottoposti a tali schemi di trattamento extracorporeo (68-70).

Per quanto concerne l'elevata prevalenza di casi di SCD nei pazienti con ESRD, piccoli trial randomizzati hanno permesso di documentare una riduzione dei casi di SCD (dal 10.4\% al $3.4 \%$ ) impiegando la terapia con carvedilolo in pazienti affetti da ESRD e affetti da cardiomiopatia dilatativa $(71,72)$.

La terapia con $\beta$-bloccanti dovrebbe essere impiegata routinariamente in pazienti affetti da CKD ed ESRD con precedenti di eventi coronari ischemici acuti non fatali (72).

\section{Diagnostica per immagini dell'ipertrofia ventricolare sinistra}

Elettrocardiografia (ECG), ecocardiografia 2D e 3D (ECHO) (Figg. 1 e 2) e risonanza magnetica cardiaca (CMRI) (Fig. 3) rappresentano i tre passaggi successivi per quantificare e stimare il grado di IVS mediante tecniche di acquisizione di immagini.

Dal punto di vista storico, l'ECG è stata la prima tecnica di diagnosi impiegata per stimare la presenza di un quadro di IVS, viste le sue caratteristiche di non-invasività, economicità e facile esecuzione e interpretazione da parte, rispettivamente, del personale infermieristico e medico.

L'ECG viene considerata un test molto poco sensibile ma sufficientemente specifico, anche se, a tutt'oggi, non sono stati stilati dei criteri definiti per la classificazione dell'IVS valutata con questa metodica (73).

D'altro canto, la CMRI rappresenta indubbiamente il gold standard per la valutazione dell'IVS, in quanto riesce a definire accuratamente la massa ventricolare sinistra, i volumi (telesistolico e telediastolico) del ventricolo sinistro, il pattern di IVS (concentrica, eccentrica, asimmetrica) e il grado di fibrosi miocardica.

Se si prendono in considerazione i pazienti sottoposti a trattamento emodialitico, l'impiego della classica ecocardiografia M-mode spesso sovrastima, rispetto alla CMRI, l'entità della massa ventricolare sinistra, ma, allo stesso tempo, non vi sono dubbi sul fatto che l'impiego della CMRI non possa essere diffuso a tutto campo, in virtù dei costi elevati e dell'impossibilità di impiegarla nei soggetti che soffrono di claustrofobia e che sono portatori di dispositivi impiantabili come pacemaker e/o defibrillatori $(74,75)$.

A causa di queste limitazioni della CMRI, l'ecocardiografia rimane ancora la tecnica di diagnostica per immagini di maggiore impiego e diffusione per la stima della massa ventrico- 


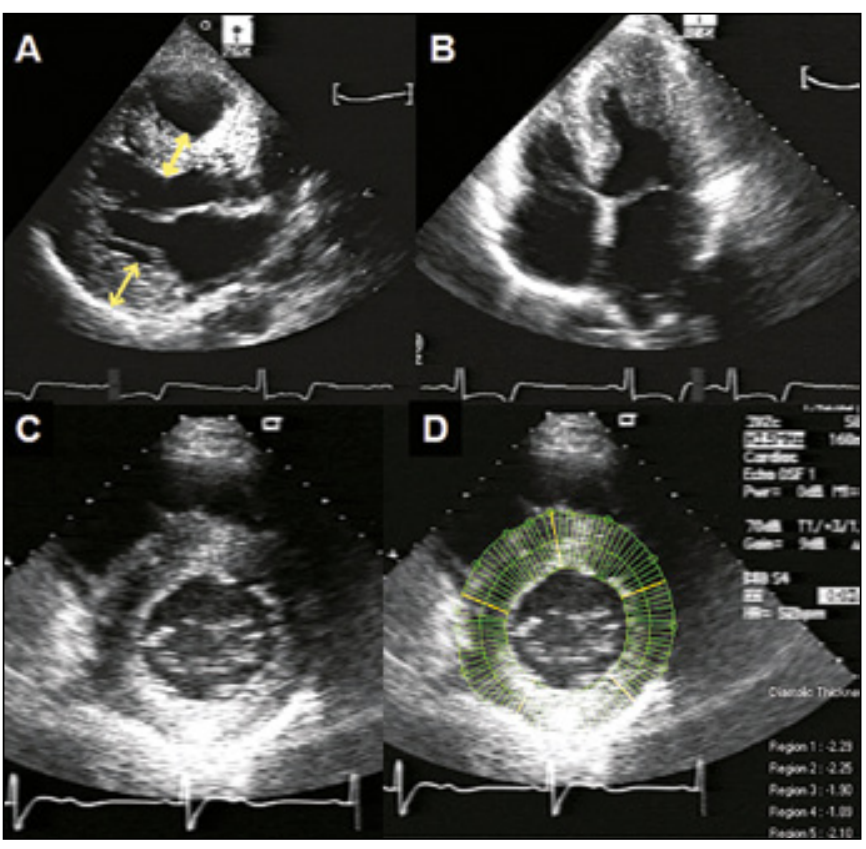

Fig. 1 - Ecocardiografia bidimensionale in grado di evidenziare la presenza di IVS in un paziente affetto da CKD allo stadio IIIb.

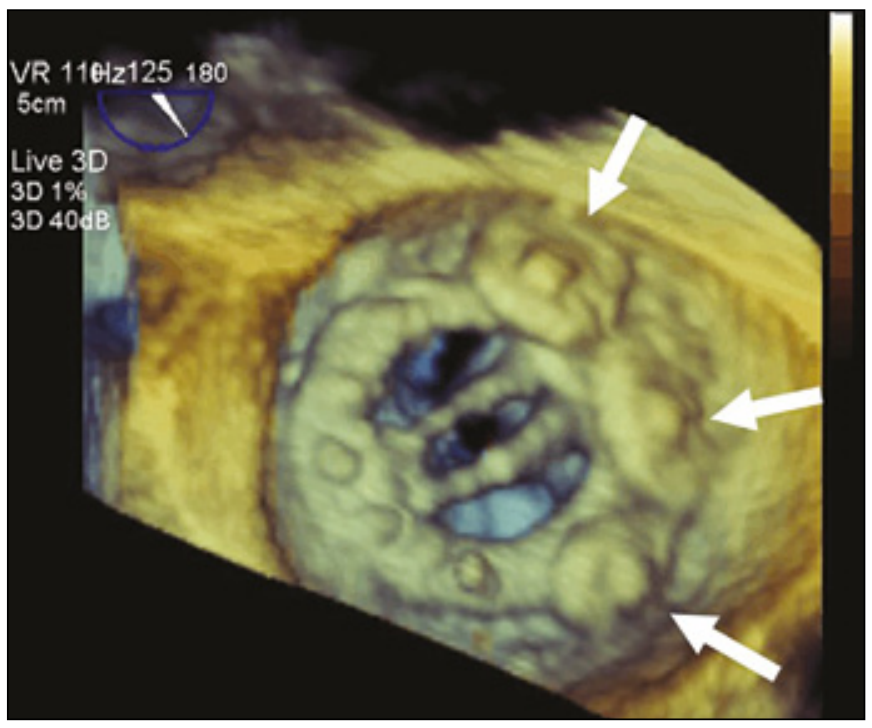

Fig. 2 - Ecocardiografia 3D in grado di rilevare la presenza di un'estesa fibrosi miocardica in un paziente sottoposto a trattamento emodialitico.

lare sinistra nella pratica clinica quotidiana, sebbene possegga delle intrinseche limitazioni. L'accuratezza dell'ecocardiografia dipende dalla tecnica utilizzata, dal timing relativo alle sedute dialitiche e dai parametri di normalizzazione e indicizzazione dei dati ricavati. Si tratta, inoltre, di una tecnica ecografica che risulta essere particolarmente operatore-dipendente, nonché fortemente legata, come le altre tecniche ecografiche, alla presenza di finestre acustiche adeguate, soprattutto nei pazienti nei quali è frequente riscontrare quadri di IVS di tipo asimmetrico $(75,76)$.

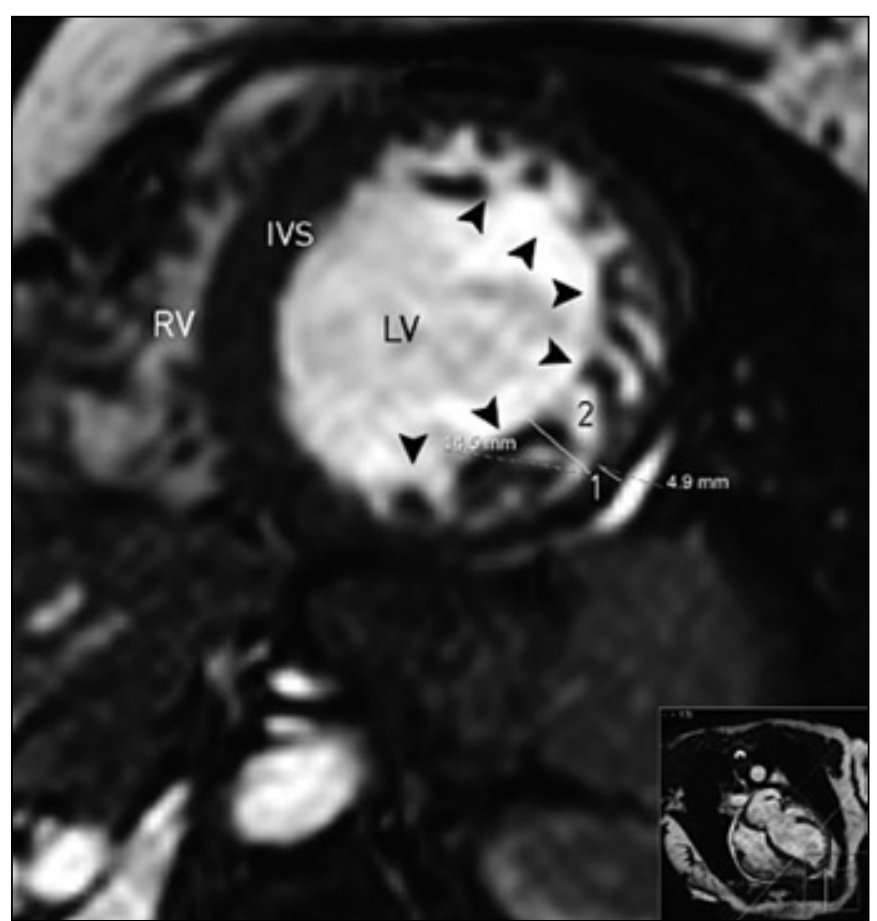

Fig. 3 - Immagine CMRI in un paziente in trattamento mediante dialisi peritoneale con IVS di tipo asimmetrico.

Un altro punto chiave per una corretta valutazione della massa ventricolare sinistra è quello relativo all'indicizzazione delle misurazioni ottenute, in quanto la massa ventricolare sinistra risulta essere proporzionale alla superficie corporea.

Purtroppo, allo stato attuale, non vi è un accordo unanime sui valori normali di massa cardiaca, motivo per cui, nei vari trial clinici, sono stati considerati diversi valori di cut-off.

Per esempio, Silverberg ha impiegato valori di cut-off pari a 125 $\mathrm{g} / \mathrm{m}^{2}$ (77), laddove Parfrey ha utilizzato i valori di cut-off considerati nello studio di Framingham (132 g/m²/ $100 \mathrm{~g} / \mathrm{m}^{2 /}+$ ), allo scopo di porre una diagnosi ecocardiografica di IVS (78). Linee Guida recenti hanno definito come valori normali di massa ventricolare sinistra i seguenti: $<45 \mathrm{~g} / \mathrm{m}$ altezza $(73$, 76) nelle donne e $<49 \mathrm{~g} / \mathrm{m}$ altezza $(73,79)$ negli uomini (80). Le tecniche ecocardiografiche $2 \mathrm{D}$ e $3 \mathrm{D}$ vengono abitualmente impiegate per la valutazione della massa ventricolare sinistra nei pazienti affetti da CKD ed ESRD, ma l'ecocardiografia $2 \mathrm{D}$ risulta essere estremamente dipendente da una definizione e una demarcazione adeguate dei bordi endocardico ed epicardico del ventricolo sinistro.

L'ecocardiografia $3 \mathrm{D}$ in real time consente una valutazione più accurata della massa ventricolare sinistra, dei volumi ventricolari e della frazione di eiezione (80) e possiede un grado di accuratezza molto simile a quello garantito dalla CMRI (81).

In conclusione, ecocardiografia e risonanza magnetica cardiaca possono essere impiegate in maniera complementare per la valutazione della fibrosi miocardica e della disfunzione diastolica del ventricolo sinistro nei pazienti affetti da CKD ed ESRD (73). La CMRI è sicuramente in grado di determinare e quantificare con maggiore precisione il grado di fibrosi miocardica, gra- 
zie anche all'enhancement fornito dall'impiego di gadolinio, mezzo di contrasto non iodato il cui utilizzo è però controindicato nei pazienti a uno stadio avanzato di CKD (82).

La CMRI rappresenta certamente la tecnica migliore per diagnosticare e quantificare l'incremento di massa ventricolare sinistra nei pazienti con malattia renale cronica ma ha, dalla sua, le limitazioni già esposte in precedenza e che la pongono, al momento, in una posizione secondaria rispetto alle più diffuse tecniche ecocardiografiche, non invasive e decisamente meno costose, anche se strettamente operatore-dipendenti.

\section{Riassunto}

La patologia cardiovascolare rappresenta la principale causa di mortalità e morbidità nei pazienti affetti da malattia renale cronica (CKD) e malattia renale cronica terminale (ESRD). La patogenesi della malattia cardiovascolare in corso di nefropatia è multifattoriale e coinvolge fattori di rischio tradizionali e fattori di rischio collegati alla malattia renale. Come ormai universalmente accettato, l'interessamento cardiaco in corso di malattia renale cronica rientra nella cosiddetta Sindrome cardio-renale di tipo 4, la cosiddetta cardiopatia uremica caratterizzata, in primo luogo, dalla presenza di ipertrofia ventricolare sinistra, disfunzione sistodiastolica del ventricolo sinistro e, negli stadi terminali, scompenso cardiaco congestizio e cardiomiopatia dilatativa.

La diagnosi di ipertrofia ventricolare sinistra (IVS) è af- fidata da un lato alle tecniche ecocardiografiche $2 \mathrm{D}$ e $3 \mathrm{D}$ e, dall'altra, a tecniche di imaging più sofisticate, come la risonanza magnetica cardiaca (CMRI).

Scopo della review è quello di effettuare un excursus riguardante l'epidemiologia, la fisiopatologia e la diagnosi dell'ipertrofia ventricolare sinistra nei pazienti affetti da malattia renale cronica.

Parole chiave: Malattia cardiovascolare, Sindrome cardio-renale di tipo 4, Ipertrofia ventricolare sinistra, Ecocardiografia

Dichiarazione di conflitto di interesse: Gli Autori dichiarano di non avere conflitto di interessi.

Contributi economici agli Autori: Gli Autori dichiarano di non avere ricevuto sponsorizzazioni economiche per la preparazione dell'articolo.

\section{Indirizzo degli Autori:}

Dr. Luca Di Lullo

U.O.C. Nefrologia e Dialisi

Ospedale L. Parodi-Delfino

Piazza Aldo Moro 1

00034 Colleferro (RM)

dilulloluca69@gmail.com

\section{Bibliografia}

1. Ronco C. The cardiorenal syndrome: basis and common ground for a multidisciplinary patient-oriented therapy. Cardiorenal Med 2011; 1: 3-4.

2. Heywood JT, Fonarow GC, Costanzo MR, Mathur VS, Wigneswaran JR, Wynne J. High prevalence of renal dysfunction and its impact on outcome in 118,465 patients hospitalized with acute decompensated heart failure: A report from the ADHERE database. J Card Fail 2007; 13 (6): 422-30.

3. Garg AX, Clark WF, Haynes RB, House AA. Moderate renal insufficiency and the risk of cardiovascular mortality: Results from the NHANES I. Kidney Int 2002; 61 (4): 1486-94.

4. Foley RN, Parfrey PS, Harnett JD, Kent GM, Murray DC, Barre PE. The prognostic importance of left ventricular geometry in uremic cardiomyopathy. J Am Soc Nephrol 1995; 5 (12): 2024-31.

5. Harnett JD, Foley RN, Kent GM, Barre PE, Murray D, Parfrey PS. Congestive heart failure in dialysis patients: Prevalence, incidence, prognosis and risk factors. Kidney Int 1995; 47 (3): 884-90.

6. U.S. Renal Data System, USRDS 2012 Annual Data Report: Atlas of End-Stage Renal Disease in the United States, National Institutes of Health, National Institute of Diabetes and Digestive and Kidney Diseases, Bethesda, MD, 2012.

7. Tonelli M, Wiebe N, Culleton B, et al. Chronic kidney disease and mortality risk: A systematic review. J Am Soc Nephrol
2006; 17 (7): 2034-47.

8. U.S. Renal Data System, USRDS 2009 Annual Data Report: Atlas of End-Stage Renal Disease in the United States, National Institutes of Health, National Institute of Diabetes and Digestive and Kidney Diseases, Bethesda, MD, 2009.

9. Go AS, Chertow GM, Fan D, McCulloch CE, Hsu CY. Chronic kidney disease and the risks of death, cardiovascular events, and hospitalization. N Engl J Med 2004; 351 (13): 1296-305.

10. Shastri S, Sarnak MJ. Cardiovascular disease and CKD: Core curriculum 2010. Am J Kidney Dis 2010; 56 (2): 399-417.

11. Stevens LA, Li S, Wang C, et al. Prevalence of CKD and comorbid illness in elderly patients in the united states: Results from the kidney early evaluation program (KEEP). Am J Kidney Dis 2010; 55 (3 Suppl. 2): S23-33.

12. Bansal N, Keane M, Delafontaine P, et al. A longitudinal study of left ventricular function and structure from CKD to ESRD: The CRIC study. Clin J Am Soc Nephrol 2013; 8 (3): 355-62.

13. Kottgen A, Russell SD, Loehr LR, et al. Reduced kidney function as a risk factor for incident heart failure: The atherosclerosis risk in communities (ARIC) study. J Am Soc Nephrol 2007; 18 (4): 1307-15.

14. Di Lullo L, Floccari F, Polito P. Right ventricular diastolic function in dialysis patients could be affected by vascular access. Nephron Clin Pract 2011; 118: c258-62.

15. Bologa RM, Levine DM, Parker TS, et al. Interleukin-6 predicts hypoalbuminemia, hypocholesterolemia, and mortality in hemodialysis patients. Am J Kidney Dis 1998; 32 (1): 107-14. 
16. Parikh SV, de Lemos JA. Biomarkers in cardiovascular disease: integrating pathophysiology into clinical practice. Am J Med Sci 2006; 332 (4): 186-97.

17. Ritz E, Wanner C. The challenge of sudden death in dialysis patients. Clin J Am Soc Nephrol 2008; 3: 920-9.

18. Gross ML, Ritz E. Hypertrophy and fibrosis in the cardiomyopathy of uremia - beyond coronary heart disease. Semin Dial 2008; 21: 308-18.

19. Ritz E. Left ventricular hypertrophy in renal disease: beyond preload and afterload. Kidney Int 2009; 75: 771-3.

20. Mominadam S, Ozkahya M, Kayikcioglu M. Interdialytic blood pressure obtained by ambulatory blood pressure measurement and left ventricular structure in hypertensive hemodialysis patients. Hemodial Int 2008; 12: 322-7.

21. Steigerwalt S, Zafar A, Mesiha N, Gardin J, Provenzano R. Role of aldosterone in left ventricular hypertrophy among AfricanAmerican patients with end-stage renal disease on hemodialysis. Am J Nephrol 2007; 27: 159-63.

22. Xu X, Hu X, Zhang P, Zhao L, Wessale JL, Bache RJ, Chen Y. Xantine oxidase inhibition with febuxostat attenuates systolic overload-induced left ventricular hypertrophy and dysfunction in mice. J Card Fail 2008; 14: 746-53.

23. Hsu S, Nagayama T, Koitabashi N, et al. Phosphodiesterase-5 inhibition blocks pressure overload-induced cardiac hypertrophy independent of the calcineurin pathway. Cardiovasc Res 2009; 81: 301-9.

24. Martin LC, Franco RJ, Gavras I, et al. Association between hypervolemia and ventricular hypertrophy in hemodialysis patients. Am J Hypertens 2004; 17: 1163-9.

25. McRae JM, Levin A, Belenkie I. The cardiovascular effects of arteriovenous fistulas in chronic kidney disease. A cause for concern ? Semin Dial 2006; 19: 349-52.

26. Nishida K, Kyoi S, Yamaguchi O, Sadoshima J, Otsu K. The role of autophagy in the heart. Cell Death Differ 2009; 16: 31-8.

27. Dorm GW 2nd. Apoptotic and non-apoptotic programmed cardiomyocyte death in ventricular remodeling. Cardiovasc Res 2009; 81: 465-73.

28. Zoccali C, Benedetto FA, Tripepi G, Mallamaci F. Cardiac consequences of hypertension in hemodialysis patients. Semin Dial 2004; 17: 299-303.

29. Sakurabayashi T, Miyazaki S, Yuasa Y, et al. L-carnitine supplementation decreases the left ventricular mass in patients undergoing hemodialysis. Circ J 2008; 72: 926-31.

30. Strózecki P, Adamowicz A, Nartowicz E, et al. Parathormon, calcium, phosphorus and left ventricular structure and function in normotensive hemodialysis patients. Ren Fail 2001; 23: 115-26.

31. Koleganova N, Piecha G, Ritz E, et al. Interstitial fibrosis and microvascular disease of the heart in uremia: amelioration by a calciomimetic. Lab Invest 2009; 89: 520-30.

32. Oberleithner H, Riethmuller C, Schillers H, Mac Gregor GA, de Wardener HE, Hausberg M. Plasma sodium-stiffens vascular endothelium and reduces nitric oxide release. Proc Natl Acad Sci U S A 2007; 104: 16281-6.

33. De Paula FM, Peixoto AJ, Pinto LV, Dorigo D, Patricio PJ, Santos SF. Clinical consequences of an individualized dialysate sodium prescription in hemodialysis patients. Kidney Int 2004; 66: 1232-8.

34. Moe S, Drueke T, Cunningham J. Definition, evaluation and classification of renal osteodistrophy: a position statement from
Kidney Disease Improving Global Outcome (KDIGO). Kidney Int 2006; 69 (11): 1945-53.

35. Moe S, Drueke TB, Block A, et al. KDIGO clinical practice guideline for the diagnosis, evaluation, prevention and treatment of chronic kidney disease-mineral and bone disorder (CKDMBD). Kidney Int 2009; 113: S1-130.

36. Burhiya R, Li S, Chen S, Mc Cullough PA, Bakris GL. Plasma parathyroid hormone level and prevalent cardiovascular disease in CKD stages 3 and 4: an analysis from the Kidney Early Evaluation Program (KEEP). Am J Kidney Dis 2009; 53 (4): S3-10.

37. Evenepoel P, Meijers B, Viaene L, et al. Fibroblast growth factor-23 in early chronic kidney disease: additional support in favor of a phosphate-centric paradigm for the pathogenesis of secondary hyperparathyroidism. Clin J Am Soc Nephrol 2010; 5 (7): $1268-76$.

38. Isakova T, Wahl P, Vargas GS. Fibroblast growth factor-23 is elevated before parathyroid hormone and phosphate in chronic kidney disease. Kidney Int 2011; 79 (12): 1370-8.

39. Faul C, Amaral AP, Oskouei B, et al. FGF23 induces left ventricular hyperthrophy. J Clin Invest 2011; 12: 4393-408.

40. Gutierrez OM, Mannstadt M, Isakova T, et al. Fibroblast growth factor 23 and mortality among patients undergoing hemodialysis. N Engl J Med 2008; 359 (6): 584-92.

41. Isakova T, Xie H, Yang W. Fibroblast growth factor 23 and risks of mortality and end-stage renal disease in patients with chronic kidney disease. JAMA 2011; 305 (23): 2432-9.

42. Liu X, Xie R, Liu S. Rat parathyroid hormone 1-34 signals through the MEK/ERK pathway to induce cardiac hypertrophy. J Int Med Res 2008; 36: 942-50.

43. Moon KH, Song IS, Yang WS, et al. Hypoalbuminemia as a risk factor for progressive left-ventricular hypertrophy in hemodialysis patients. Am J Nephrol 2000; 20: 396-401.

44. Chmielewski M, Carrero JJ, Stenvinkel P, Lindholm B. Metabolic abnormalities in chronic kidney disease that contribute to cardiovascular disease, and nutritional initiatives that may diminish the risk. Curr Opin Lipidol 2009; 20: 3-9.

45. Wolf M, Shah A, Gutierrez O, et al. Vitamin D levels and early mortality among incident hemodialysis patients. Kidney Int 2007; 72: 1004-13.

46. Mehrotra R, Kermah D, Budoff M, et al. Hypovitaminosis D in chronic kidney disease. Clin J Am Soc Nephrol 2008; 3: 1144-51.

47. Testa A, Mallamaci F, Benedetto F, et al. Vitamin D Receptor (VDR) Gene Polymorphism Is Associated With Left Ventricular (LV) Mass and Predicts Left Ventricular Hypertrophy (LVH) Progression in End-Stage Renal Disease (ESRD) Patients. J Bone Miner Res 2010; 25: 313-9.

48. El-Shehaby AM, El-Khatib MM, Marzouk S, Battah AA. Relationship of BsmI polymorphism of vitamin D receptor gene with left ventricular hypertrophy and atherosclerosis in hemodialysis patients. Scand J Clin Lab Investig 2013; 73: 75-81.

49. Zoccali C, Benedetto FA, Mallamaci F, et al. Left ventricular mass monitoring in the follow-up of dialysis patients: Prognostic value of left ventricular hypertrophy progression. Kidney Int 2004; 65: 1492-8.

50. London GM, Pannier B, Guerin AP, et al. Alterations of left ventricular hypertrophy in and survival of patients receiving hemodialysis: follow-up of an interventional study. J Am Soc Nephrol 
2001; 12: 2759-67.

51. Krane V, Winkler K, Drechsler C, Lilienthal J, Marz W, Wanner C. Effect of atorvastatin on inflammation and outcome in patients with type 2 diabetes mellitus on hemodialysis. Kidney Int 2008; 74: 1461-7.

52. Aoki J, Ikari Y, Nakajima H, et al. Clinical and pathologic characteristics of dilated cardiomyopathy in hemodialysis patients. Kidney Int 2005; 67: 333-40.

53. Paoletti E, Bellino D, Cassottana P, Rolla D, Cannella G. Left ventricular hypertrophy in nondiabetic predialysis CKD. Am J Kidney Dis 2005; 46: 320-7.

54. McMahon LP, Roger SD, Levin A. Development, prevention, and potential reversal of left ventricular hypertrophy in chronic kidney disease. J Am Soc Nephrol 2004; 15: 1640-7.

55. Culleton BF, Walsh M, Klarenbach SW, et al. Effect of frequent nocturnal hemodialysis vs conventional hemodialysis on left ventricular mass and quality of life: A randomized controlled trial. JAMA 2007; 298: 1291-9.

56. Foley RN, Parfrey PS, Kent GM, Harnett JD, Murray DC, Barre $\mathrm{PE}$. Serial change in echocardiographic parameters and cardiac failure in end-stage renal disease. J Am Soc Nephrol 2000; 11: 912-6.

57. Covic A, Mardare NG, Ardeleanu S, Prisada O, Gusbeth-Tatomir P, Goldsmith DJ. Serial echocardiographic changes in patients on hemodialysis: An evaluation of guideline implementation. J Nephrol 2006; 19: 783-93.

58. Marchais SJ, Metivier F, Guerin AP, London GM. Association of hyperphosphataemia with haemodynamic disturbances in end-stage renal disease. Nephrol Dial Transplant 1999; 14: 2178-83.

59. Galetta F, Cupisti A, Franzoni F, et al. Left ventricular function and calcium phosphate plasma levels in uraemic patients. J Intern Med 2005; 258: 378-84.

60. Parfrey PS, Lauve M, Latremouille-Viau D, Lefebvre P. Erythropoietin therapy and left ventricular mass index in CKD and ESRD patients: A meta-analysis. Clin J Am Soc Nephrol 2009; 4: 755-62.

61. Chen HH, Tarng DC, Lee KF, Wu CY, Chen YC. Epoetin alfa and darbepoetin alfa: Effects on ventricular hypertrophy in patients with chronic kidney disease. J Nephrol 2008; 21: 543-9.

62. Ayus JC, Go AS, Valderrabano F, et al. Effects of erythropoietin on left ventricular hypertrophy in adults with severe chronic renal failure and hemoglobin $10 \mathrm{~g} / \mathrm{dL}$. Kidney Int 2005; 68: 788-95.

63. Mattioli AV, Zennaro M, Bonatti S, Bonetti L, Mattioli G. Regression of left ventricular hypertrophy and improvement of diastolic function in hypertensive patients treated with telmisartan. Int J Cardiol 2004; 97: 383-8.

64. Devereux RB, Palmieri V, Liu JE, et al. Progressive hypertrophy regression with sustained pressure reduction in hypertension: The Losartan Intervention For Endpoint Reduction study. J Hypertens 2002; 20: 1445-50.

65. Jula AM, Karanko HM. Effects on left ventricular hypertrophy of long-term nonpharmacological treatment with sodium restriction in mild-to-moderate essential hypertension. Circulation 1994; 89: 1023-31.

66. Charra B, Chazot C. Volume control, blood pressure and cardiovascular function. Lessons from hemodialysis treatment. Nephron Physiol 2003; 93: 94-101.
67. Achinger SG, Ayus JC. The role of vitamin D in left ventricular hypertrophy and cardiac function. Kidney Int 2005; 68: S37-42.

68. Ly J, Chan CT. Impact of augmenting dialysis frequency and duration on cardiovascular function. ASAIO J 2006; 52 : e11-4.

69. Fagugli RM, Pasini P, Pasticci F, Ciao G, Cicconi B, Buoncristiani U. Effects of short daily hemodialysis and extended standard hemodialysis on blood pressure and cardiac hypertrophy: A comparative study. J Nephrol 2006; 19: 77-83.

70. Weinreich T, De los Rios T, Gauly A, Passlick-Deetjen J. Effects of an increase in time vs. frequency on cardiovascular parameters in chronic hemodialysis patients. Clin Nephrol 2006; 66: 433-9.

71. Kong CH, Farrington K. Determinants of left ventricular hypertrophy and its progression in high-flux haemodialysis. Blood Purif 2003; 21: 163-9.

72. Cice G, Ferrara L, D'Andrea A, et al. Carvedilol increases twoyear survivalin dialysis patients with dilated cardiomyopathy: A prospective, placebo-controlled trial. J Am Coll Cardiol 2003; 41: 1438-44.

73. Pewsner D, Juni P, Egger M, Battaglia M, Sundstrom J, Bachmann LM. Accuracy of electrocardiography in diagnosis of left ventricular hypertrophy in arterial hypertension: systematic review. BMJ 2007; 335: 711.

74. Stewart GA, Foster J, Cowan M, et al. Echocardiography overestimates left ventricular mass in hemodialysis patients relative to magnetic resonance imaging. Kidney Int 1999; 56 : 2248-53.

75. Mark PB, Patel RK, Jardine AG. Are we overestimating left ventricular abnormalities in end-stage renal disease? Nephrol Dial Transplant 2007; 22: 1815-9.

76. Zoccali C, Benedetto FA, Mallamaci F, et al. Prognostic impact of the indexation of left ventricular mass in patients undergoing dialysis. J Am Soc Nephrol 2001; 12: 2768-74.

77. Silberberg JS, Barre PE, Prichard SS, Sniderman AD. Impact of left ventricular hypertrophy on survival in end-stage renal disease. Kidney Int 1989; 36: 286-90.

78. Parfrey PS, Foley RN, Harnett JD, Kent GM, Murray DC, Barre PE. Outcome and risk factors for left ventricular disorders in chronic uraemia. Nephrol Dial Transplant 1996; 11: 1277-85.

79. Ritz E, Bommer J. Cardiovascular problems on hemodialysis: current deficits and potential improvement. Clin J Am Soc Nephrol 2009; 4: S71-8.

80. Lang RM, Bierig M, Devereux RB, et al. Recommendations for chamber quantification: a report from the American Society of Echocardiography's Guidelines and Standards Committee and the Chamber Quantification Writing Group, developed in conjunction with the European Association of Echocardiography, a branch of the European Society of Cardiology. J Am Soc Echocardiogr 2005; 18: 1440-63.

81. Takeuchi M, Nishikage T, Mor-Avi V, et al. Measurement of left ventricular mass by real-time three-dimensional echocardiography: Validation against magnetic resonance and comparison with two-dimensional and m-mode measurements. J Am Soc Echocardiogr 2008; 21: 1001-5.

82. Kribben A, Witzke O, Hillen U, Barkhausen J, Daul AE, Erbel R. Nephrogenic systemic fibrosis: Pathogenesis, diagnosis, and therapy. J Am Coll Cardiol 2009; 53: 1621-8. 